\title{
A critical assessment of two-body and three-body interactions in water
}

\author{
Gregory R. Medders, ${ }^{1, a)}$ Volodymyr Babin, ${ }^{1, a)}$ and Francesco Paesani, b) \\ Department of Chemistry and Biochemistry, University of California, San Diego, La Jolla, \\ CA 92103
}

(Dated: 1 November 2018)

The microscopic behavior of water under different conditions and in different environments remains the subject of intense debate. A great number of the controversies arises due to the contradictory predictions obtained within different theoretical models. Relative to conclusions derived from force fields or density functional theory, there is comparably less room to dispute highly-correlated electronic structure calculations. Unfortunately, such ab initio calculations are severely limited by system size. In this study, a detailed analysis of the two- and three-body water interactions evaluated at the CCSD(T) level is carried out to quantitatively assess the accuracy of several force fields, density functional theory, and ab initio-based interaction potentials that are commonly used in molecular simulations. Based on this analysis, a new model, HBB2-pol, is introduced which is capable of accurately mapping $\operatorname{CCSD}(\mathrm{T})$ results for water dimers and trimers into an efficient analytical function. The accuracy of HBB2-pol is further established through comparison with the experimentally determined second and third virial coefficients.

\section{INTRODUCTION}

Connecting small clusters of water and the condensed phases of water through a single molecular model has been a long sought-after but so-far unachieved goal. The challenges involved in the pursuit of this goal are numerous. For example, at the cluster level, the BornOppenheimer energies of topologically distinct isomers of the water hexamer differ by less than $1 \mathrm{kcal} / \mathrm{mol}^{1} 3$, indicating that highly-correlated electronic structure calculations are required to quantitatively determine the energy order of these isomers. In this regard, a faithful description of molecular flexibility appears to be particularly important. $\stackrel{3}{3}$ Furthermore, it has also been shown that nuclear quantum-mechanical effects can impact the structural, thermodynamic, and dynamical properties of both clusters and bulk phases of water.4 7 The explicit inclusion of nuclear quantum effects in simulations exacerbates the computational expense of a model, providing additional strain on the ability to obtain statistically meaningful results.

The majority of water simulations rely on force fields, which are built upon the many-body expansion of the interaction energy,

$$
\begin{aligned}
& E(1, \ldots, N)=\sum_{i}^{N} V^{1 B}(i)+\sum_{i<j}^{N} V^{2 B}(i, j) \\
& +\sum_{i<j<k}^{N} V^{3 B}(i, j, k)+\cdots+V^{N B}(1, \ldots, N) .
\end{aligned}
$$

Here, $V^{1 B}(i)=E(i)-E_{e q}(i)$ is the one-body (1B) potential, which describes the energy required to deform an individual molecule from its equilibrium geometry.

\footnotetext{
a) Contributed equally to this work

b) Electronic mail: fpaesani@ucsd.edu
}

In common force-fields, the 1B interactions include all bonded terms (i.e., stretches, bends, and torsions). For systems, such as water, that are easily reduced to distinct molecules, a $1 \mathrm{~B}$ configuration is typically referred to as a "monomer", and groups of $2,3, \ldots, N$ interacting monomers are then termed "dimers", "trimers", ..., "N-mers". In Eq. 1, higher-order interactions are defined recursively through the lower-order terms. For instance, the two-body $(2 \mathrm{~B})$ interaction is expressed as

$$
V^{2 B}=E(1,2)-\sum_{i=1}^{2} E(i)
$$

where $E(1,2)$ is the dimer energy. Similarly, the threebody (3B) interaction is

$$
V^{3 B}=E(1,2,3)-\sum_{i<j}^{3} E(i, j)+\sum_{i=1}^{3} E(i)
$$

with $E(1,2,3)$ being the trimer energy. Common force fields are pairwise additive, meaning that three-body and higher interactions are neglected.

If it converges quickly, the many-body expansion represents a powerful approach to studying condensed phases as it allows for the energy of an $N$-molecule system to be expressed as a sum of lower-order interactions that can in principle be calculated with high accuracy. Recently, a detailed study of the convergence of the many-body expansion for water based on the analysis of small clusters was performed using coupled cluster theory with single, double, and perturbative triple excitations $[\mathrm{CCSD}(\mathrm{T})]$ and large basis sets ${ }^{3}$ Consistent with previous observations 9 , it was determined that, although two-body interactions dominate the expansion, the threebody term can contribute up to $30 \%$ of the total energy of the water hexamer. An estimate of the relative magnitudes of the many-body terms in liquid was obtained through an RIMP2 analysis of the 21-mer, for which twobody interactions were found to contribute $75-80 \%$ of the 
total interaction energy and three-body interactions comprised 15-20\%! ${ }^{[16}$ For both the water hexamer and the 21mer, higher-order terms contribute less than $5 \%$ of the total interaction energy. It should be noted that, while quickly converging for water, the many-body expansion has been shown to converge slowly and with marked oscillatory behavior for other systems. 17

In this study, the accuracy of several force fields, density functional theory (DFT), and $a b$ initio potentials in reproducing the two- and three-body water interactions is assessed through a detailed comparison with data obtained at the CCSD(T) level of theory (Section II). Based on this analysis, a new ab initio water model, HBB2-pol, is then introduced in Section III. In Section IV] we show that HBB2-pol accurately maps the $\operatorname{CCSD}(\mathrm{T})$ results for both the $2 \mathrm{~B}$ and $3 \mathrm{~B}$ interactions into an efficient analytical functional form and predicts the second and third virial coefficients in excellent agreement with the available experimental data. A summary is given in Section V.

\section{ANALYSIS OF TWO- AND THREE-BODY WATER INTERACTIONS}

\section{A. Water models}

The many-body expansion provides the underlying basis for common classical force fields. In most cases, including the widely-used TIP4P and SPC families ${ }^{18 \mid 19}$, pairwise additivity is assumed, with three- and higherbody interactions being "encoded" into the effective twobody contributions. In addition, the majority of these models treat the water molecules as rigid monomers (i.e., the $1 \mathrm{~B}$ interactions are set to zero), with only few quantum water models, notably as q-TIP4P/f and qSPC/Fw, 20|21 allowing for molecular flexibility. Nevertheless, pairwise force fields have been surprisingly successful at reproducing, at least qualitatively, the properties of water in homogeneous environments. ${ }^{22}$ However, such force fields are expected to be inherently limited in their ability to model the microscopic behavior of aqueous interfaces, water confined at the nanoscale, and clusters, whose properties are sensitive to the detailed interplay of 1B, 2B, 3B, and higher-body interactions. 16

Recent work has focused on improving empirical models through inclusion of three-body interactions, leading to the development of the E3B model.2324 Although the inclusion of explicit 3B interactions greatly improves the accuracy of the E3B model relative to pairwise force fields, the use of rigid water monomers and empirical parameterization necessarily misses some of the fundamental properties of the many-body expansion. For example, recent E3B simulations of the isomeric equilibria of the water hexamer have led to predictions that are markedly different from $a b$ initio calculations. Specifically, the prism structure, which corresponds to the energetically lowest-lying isomer at the MP2 and CCSD(T) levels of theory,,$^{[2] 3}$ is unstable in the E3B calculations.

Since non-pairwise additive intermolecular interactions arise primarily from electronic polarization at long distances, several methods have been proposed to incorporate this effect into the framework of classical force fields. ${ }^{26}$ One common approach is the Applequist polarizable point dipole model, 27 which was elaborated upon by Thole to address the so-called polarization catastrophe ${ }^{28}$ Thole-type polarizable force fields for water include TTM3-F 29 , TTM4-F 30 , and AMOEBA ${ }^{31}$ models.

Among methods that attempt to solve directly the many-body problem from "first principles", semiempirical models represent an attractive alternative due to their computational efficiency. Semiempirical models such as PM3 32 and PM3-MAIS ${ }^{33}$ were derived within the MNDO scheme and differ primarily in the form of the core-core repulsion as well as in the precise values of their adjustable parameters. These models were parametrized either by fitting experimental data for a wide variety of systems (PM3) or $a b$ initio reference data in the case of PM3-MAIS. Due to the use of a minimal basis and the explicit neglect of correlation, semiempirical methods are particularly limited in their ability to describe non-bonded interactions. This deficiency has been addressed by the SCP-NDDO model, which augments traditional semiempirical methods with classical polarization. 34 SCP-NDDO has shown success in modeling water clusters and has recently been extended to simulations of bulk properties. 35

Different DFT methods has also been extensively used to the study of condensed phases, primarily through the use of GGA functionals such as BLYP $36[37$ and PBE $\$ 38$ However, common density functionals are by construction limited in their ability to describe weakly interacting van der Waals complexes. One attempt to address this problem involves the addition of a dispersion correction to the energy through the $C_{6} / R^{6}$ term, where the $C_{6}$ parameters are atom and basis-set specific 3940 . These "DFT-D" models have successfully described systems such as the solvation of iodide in water $\stackrel{41}{\text {, but are }}$ limited by the need to develop parameters for each functional/basis set ${ }^{42}$ Furthermore, because the correction is pairwise additive, it neglects higher-body dispersion contributions. Recent work to address this limitation has been reported. 43

A promising alternative to the pairwise DFT-D correction is represented by the non-local van der Waals (nlvdW) functionals. ${ }^{44}$ These nl-vdW functionals utilize the electron density to define a non-local correlation contribution to the exchange-correlation functional, leading to a consistent description of both short-range and longrange interactions. Since no atomic or basis-set dependent parameters are required to describe the dispersion interaction due to the explicit dependence of the nonlocal correlation on the electron density, nl-vdW functionals, in principle, require minimal parameterization and are system-independent. In practice, great care must be taken to avoid double counting of correlation effects in 
the combination of semi-local and non-local terms. Van der Waals density functionals have recently been applied to the study of liquid water $\underline{47}$ and ice 48 .

One final class of models is represented by the $a b i n i$ tio-based interaction potentials. These models are built upon a rigorous treatment of the many-body expansion of interactions and are characterized by having a functional form that is sufficiently flexible to accurately map high-quality $a b$ initio reference data. Examples of such models are DPP2 ${ }^{12}$ CC-pol, $\stackrel{49 \mid 50}{4}$ and WHBB! ${ }^{[1]}$ DPP2 and CC-pol are restricted to the rigid, vibrationally averaged monomer geometries, while WHBB uses permutationally invariant polynomials to represent the flexible monomer $2 \mathrm{~B}$ and $3 \mathrm{~B}$ potential energy surfaces (PESs). Such $a b$ initio-based interaction potentials are quite computationally demanding and are most commonly used in calculations for gas phase systems ${ }^{6}$, although bulk properties have been obtained from classical simulations with CC-po $\sqrt{49152}$ and DPP2 2 . Very recently, a flexible version of CC-pol has been developed, CC-pol-8s $f$, and the effects of flexibility on the dimer vibrational-rotation-tunneling (VRT) spectra have been characterized. ${ }^{53}$ It was found that both CC-pol-8s $f$ and HBB2 (the 2B potential of WHBB) reproduce the experimental VRT spectra "about equally well"

\section{B. Comparison to $\operatorname{CCSD}(\mathrm{T})$}

Here, we assess the ability of the models presented in Section $\amalg \mathrm{A}$ to describe the $2 \mathrm{~B}$ and $3 \mathrm{~B}$ water interactions. Roughly $14002 \mathrm{~B}$ interactions and $5003 \mathrm{~B}$ interactions were evaluated at the $\operatorname{CCSD}(\mathrm{T}) /$ aug-cc-pVTZ leve ${ }^{54 / 55}$ and corrected for the basis set superposition error (BSSE) using the counterpoise method. ${ }^{[56}$ These (flexible) molecular configurations were extracted from 1) classical molecular dynamics (MD) simulations of hexamers at $\mathrm{T} \leq 30 \mathrm{~K}$ using the WHBB potential, 2) classical MD simulations of ice $\mathrm{I}_{\mathrm{h}}$ carried out with TTM3-F at $50 \mathrm{~K}$, and 3) classical MD simulations of bulk water at $298 \mathrm{~K}$ and experimental density using TTM3-F. Hereafter, these configurations are referred to as "lowenergy" configurations. For the analysis of E3B, the $\mathrm{CCSD}(\mathrm{T})$ reference interaction energies were recomputed for "rigidified" molecules corresponding to the flexible configurations that were used in the comparison of the other models. All DFT energies were computed using the aug-def2-TZVPP basis ${ }^{55157}$ with the exception of BLYP$\mathrm{D}$, for which the TZVPP basis was used as in the original parametrization of the model ${ }^{39 / 40 \mid 58} \mathrm{MP} 2$ energies were computed with the aug-cc-pVTZ basis, and both DFT and MP2 interactions were corrected for BSSE. All $a b$ initio calculations were performed using the freelyavailable $a b$ initio package ORCA ${ }^{59}$. PM3 and PM3MAIS energies were calculated using the AMBER/SQM semi-empirical package ${ }^{60}$, while the SCP-NDDO energies were obtained using CP2K ${ }^{6162}$ A linear regression analysis for the data presented in Figures 1 and 2, as well as root mean square error with respect to $\operatorname{CCSD}(\mathrm{T})$ data, are presented in the supporting material.

Figures 1 and 2 show correlation plots for the $2 \mathrm{~B}$ and 3B interactions calculated for all models described in Section II A relative to the CCSD(T)/aug-cc-pVTZ energies. While most empirical pairwise force fields implicitly include nuclear quantum effects, models such as q$\mathrm{TIP} 4 \mathrm{P} / \mathrm{f}$ and $\mathrm{q}-\mathrm{SPC} / \mathrm{Fw}$ were specifically parameterized for quantum simulations and, therefore, presumably provide an approximation to the actual Born-Oppenheimer PES.20121 As can be seen from Figure 1, q-TIP4P/f deviates substantially from the $\operatorname{CCSD}(\mathrm{T}) 2 \mathrm{~B}$ potential energy surface to compensate for the neglect of higherorder interactions (Figure 22). Force fields that account for higher-order terms generally provide a more accurate description of the $2 \mathrm{~B}$ interactions than effective pairwise models. In this context, while E3B and TTM3-F/TTM4F/AMOEBA treat higher-order interactions using different schemes, all four models give $2 \mathrm{~B}$ interactions that are in closer agreement with the $\operatorname{CCSD}(\mathrm{T})$ results than the effective pairwise models. It is interesting to note that E3B, which does not not explicitly include induction and was not parameterized using ab initio data, describes the 3B contributions energies reasonably well.

The three polarizable models considered in this study (TTM3-F, TTM4-F, and AMOEBA) differ in the way they describe the variation of the molecular charge distribution. As an isolated monomer deforms, the molecular dipole moment varies in a "nonlinear" fashion with respect to the intramolecular coordinates, resulting in a "nonlinear dipole moment surface" (DMS) ${ }^{63}$ In TTM4-F, the first-order changes of the DMS are fit to electric multipoles and polarizabilites calculated at the MP2 level. The intramolecular dependence of the atomic charges in TTM3-F was instead motivated by the observation that, while the gas phase monomer charges decrease during the homolytic dissociation, a water molecule in the condensed phase dissociates into charged ions. This argument was used to justify an empirical correction to $a b$ initio-derived values, giving rise to effective charges that increase as the monomer geometry departs from equilibrium. By contrast, although AMOEBA takes into account intramolecular flexibility, the monomer charges are geometry independent $\underline{31}$ Interestingly, while the accurate monomer DMS has been reported to be essential to reproducing the solvated monomer geometry, $[63$ the three-body interaction of AMOEBA is only slightly less accurate than TTM4-F, with RMS errors of 0.22 and $0.09 \mathrm{kcal} / \mathrm{mol}$ respectively. It is also important to mention that, unlike in TTM3-F, in both AMOEBA and TTM4-F the molecular polarzability is anisotropic. It is unclear whether the inaccuracy observed in the TTM3-F 3B energies arises from its use of effective charges, isotropic molecular polarizability or both.

Among the semiempirical methods, PM3 was fitted to a wide range of experimental and $a b$ initio data, while PM3-MAIS and SCP-NDDO were both fitted to $a b$ ini- 

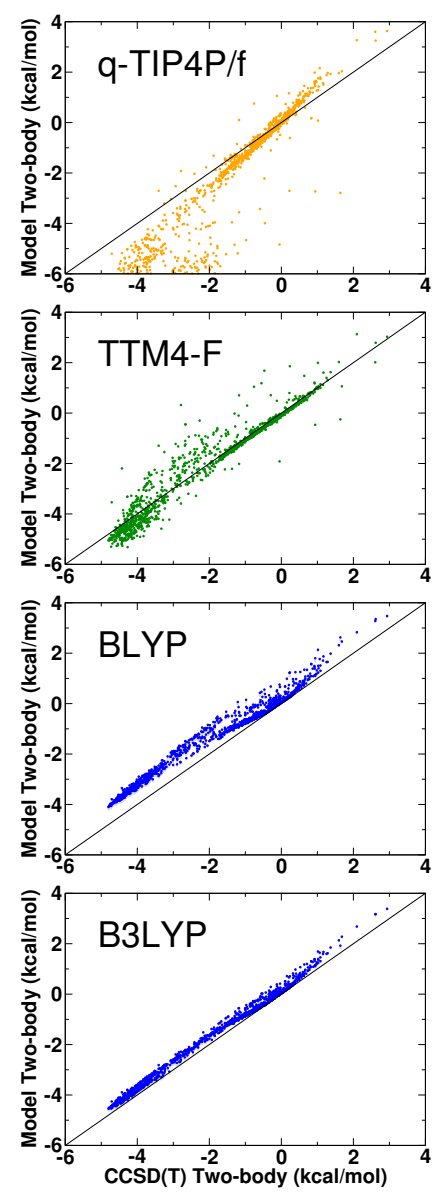
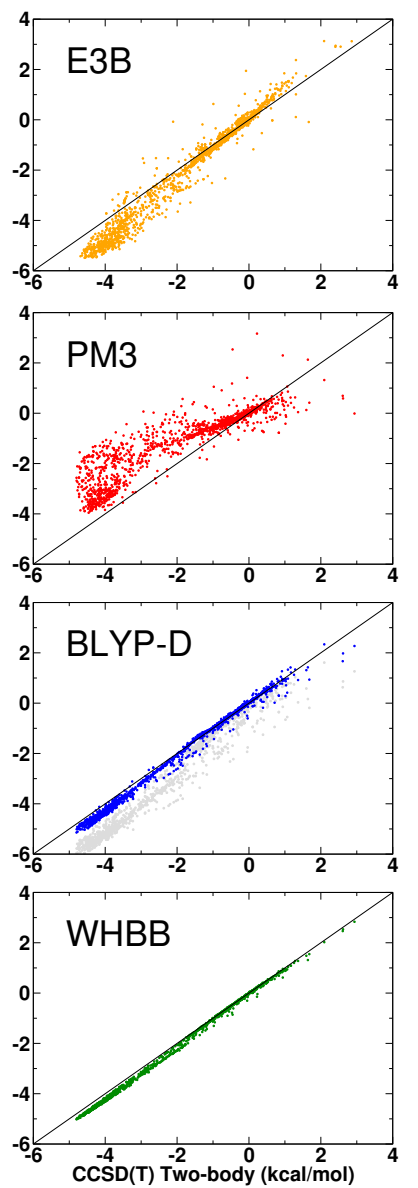
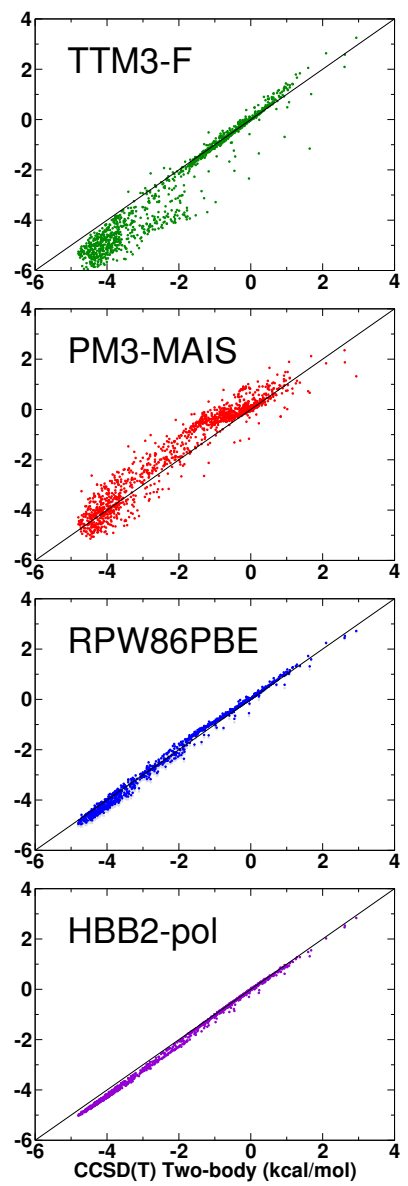
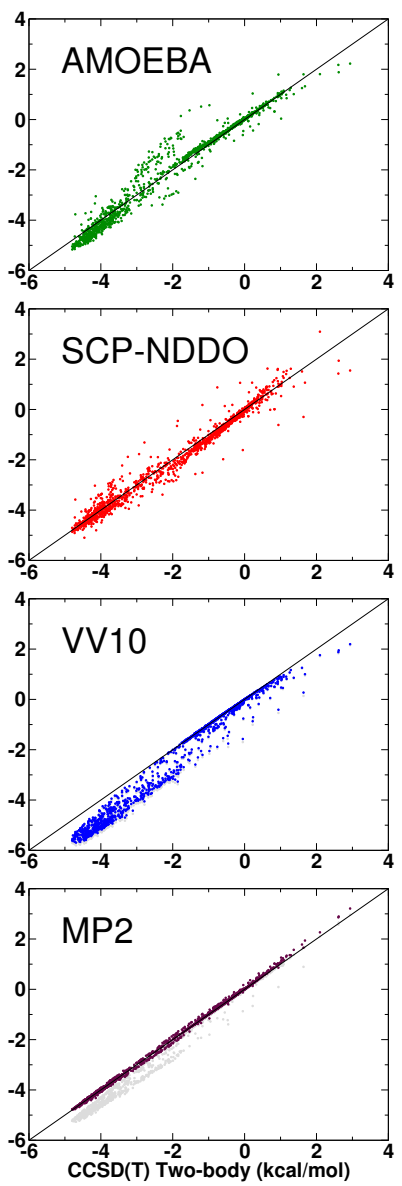

FIG. 1. Correlation plots for the 2B interactions. Plotted on the x-axes is the BSSE-corrected CCSD(T)/aug-cc-pVTZ energies. On the y-axes are the energies for each model. Empirically parametrized models are in orange, polarizable models in green, semiempirical methods in red, DFT methods in blue, and MP2 in maroon. For DFT and MP2, the colored dots are BSSEcorrected energies, while gray dots are BSSE-uncorrected energies. The new ab initio-based model, HBB2-pol, is in violet.

tio reference data of water clusters. It is therefore not surprising that the $2 \mathrm{~B}$ interactions of PM3-MAIS and SCP-NDDO are in better agreement with the CCSD $(\mathrm{T})$ data than PM3. It is interesting, however, that SCPNDDO shows much tighter correlation to the $a b$ initio data than PM3-MAIS, even though the latter uses almost twice as many adjustable parameters as SCPNDDO. While the two MNDO-type semiempirical methods display significant deficiencies in describing the $3 \mathrm{~B}$ interactions, SCP-NDDO reproduces the $\operatorname{CCSD}(\mathrm{T})$ data quite accurately. These results suggest that the addition of classical polarization, as implemented in SCP-NDDO, can allow semiempirical methods to accurately describe intermolecular interactions without requiring extensive reparametrizations of the core-core terms.

At the 2B level, the GGA density functionals differ appreciably from the $\operatorname{CCSD}(\mathrm{T})$ results (see Supporting Information for PBE and PBE0 results), with BLYP systematically underestimating the interaction strength. The inclusion of the dispersion correction in BLYP-D improves the agreement with the $\operatorname{CCSD}(\mathrm{T})$ values for the 2B interactions. However, although DFT is less sensitive to basis set incompleteness than wavefunction methods, the absence of diffuse functions in the BLYP-D basis results in a large BSSE correction (see figure 1, where blue circles give the BSSE-corrected interaction and gray circles the BSSE-uncorrected interaction). Indeed, BSSE is so small for BLYP, B3LYP, RPW86PBE, and VV10 that it is barely visible in Figures 1 and 2 . While BLYP-D can accurately describe the $2 \mathrm{~B}$ interactions when a sufficiently large basis set is used or the energy values are corrected for BSSE, how to balance these factors in condensed phase simulations is not straightforward and is the subject of ongoing research. $\underline{4264}$

While the use of hybrid functionals, such as B3LYP $\frac{65166}{}$, results in a much tighter correlation to the $\operatorname{CCSD}(\mathrm{T})$ data than GGA functionals, B3LYP nonetheless inherently suffers from inadequate treatment of dispersion interactions, which leads to an incorrect longrange behavior 67 Among recent nl-vdW functionals, VV10 appears to over-correct its parent functional, RPW86PBE, leading to over bound 2B interactions. All DFT methods perform reasonably well for the $3 \mathrm{~B}$ interactions. It is important to note that, because the dis- 

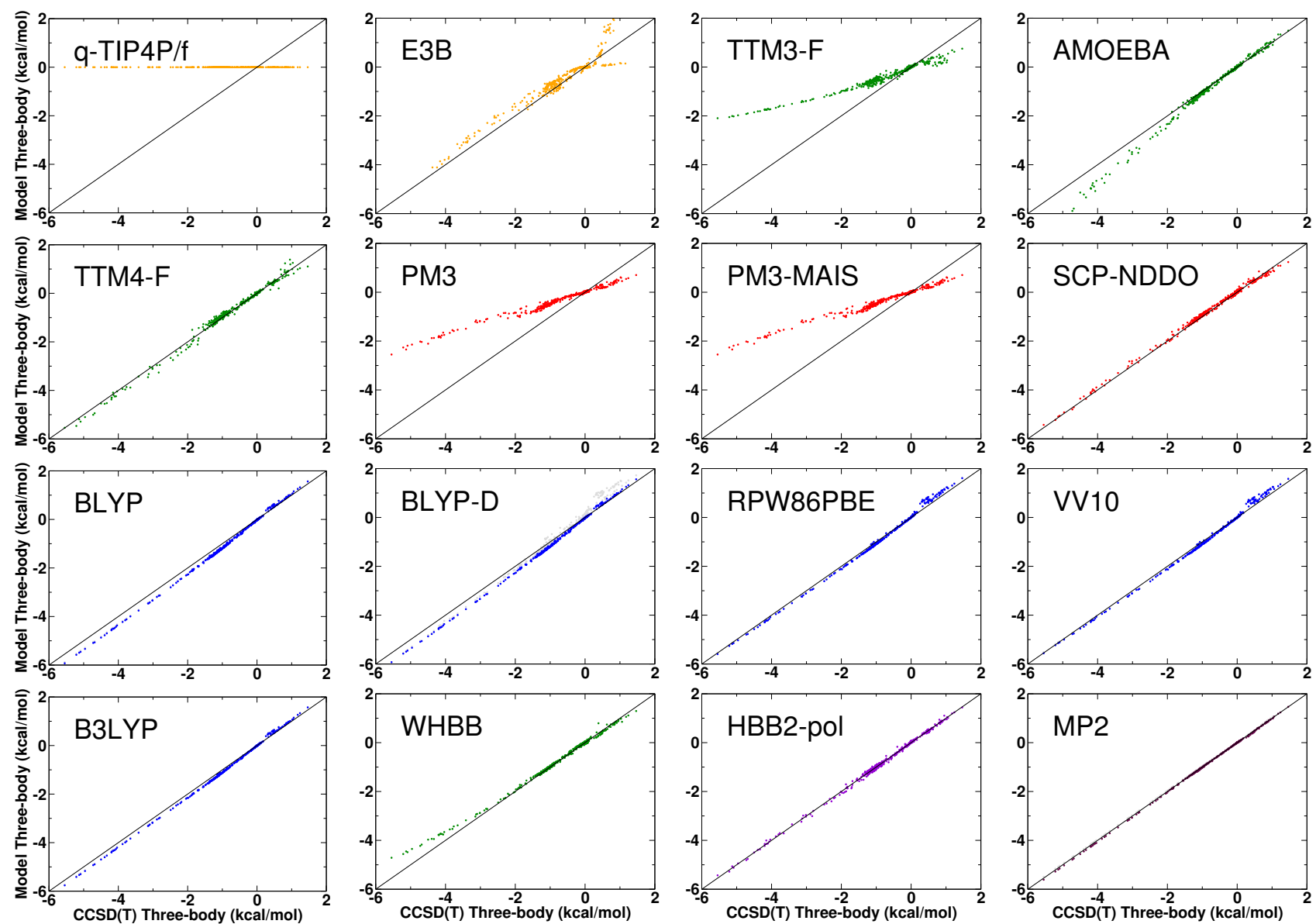

FIG. 2. Correlation plots for the 3B interactions. Plotted on the x-axes is the CCSD(T)/aug-cc-pVTZ energies corrected for BSSE. On the y-axes are the 3B energies for each model. The color scheme is the same as in Figure 1

persion correction is pair additive, BLYP and BLYP-D provide identical 3B interactions. By contrast, nl-vdW functionals include a three-body dispersion correction, although this is almost negligible for VV10 (see Supporting Information). MP2 agrees well with $\operatorname{CCSD}(\mathrm{T})$, with an RMS of 0.03 and $0.02 \mathrm{kcal} / \mathrm{mol}$ for the $2 \mathrm{~B}$ and $3 \mathrm{~B}$ interactions, respectively. Consistent with previous observations, the magnitude of BSSE is much smaller for $3 \mathrm{~B}$ than $2 \mathrm{~B}$ interactions. 68

With the exception of MP2, WHBB provides the lowest RMS for the $2 \mathrm{~B}$ interactions. WHBB employs a permutationally invariant polynomial with 5227 coefficients that were fit to reproduce $\sim 30000 \mathrm{CCSD}(\mathrm{T}) /$ augcc-pVTZ 2B interactions. To account for basis-set truncation, the reference $2 \mathrm{~B}$ interactions were chosen as weighted averages of BSSE-corrected and BSSEuncorrected $\operatorname{CCSD}(\mathrm{T})$ interactions $[69$. Since both WHBB and CC-pol reproduce the VRT spectrum of the water dimer with comparable accuracy, ${ }_{[53}^{[5}$ a similar agreement with the $\operatorname{CSSD}(\mathrm{T})$ data at the $2 \mathrm{~B}$ level is also expected for CC-pol. The agreement of WHBB with the $\operatorname{CCSD}(\mathrm{T})$ values for the $3 \mathrm{~B}$ interactions is less satisfactory, with WHBB increasingly underestimating the energies of the lowest-lying trimers. Results for the HBB2-pol model will be discussed in the following sections.

\section{METHODS}

Due to its rapid convergence for water, the many-body expansion of interaction energies provides a viable way to "scale up" the $\operatorname{CCSD}(\mathrm{T})$ level of accuracy to a large number of molecules. Furthermore, by accurately fitting the $1 \mathrm{~B}, 2 \mathrm{~B}$, and $3 \mathrm{~B}$ interactions into a relatively inexpensive function, simulations of condensed phases at an effective $\operatorname{CCSD}(\mathrm{T})$ level of accuracy become feasible. For flexible monomers, the most sophisticated effort along these lines, WHBB,$[1]$ has indisputably proven this concept. However, WHBB is not directly applicable to bulk phase simulations due to its prohibitively expensive 3B term. Motivated by this observation, this section reports the development of a new model, HBB2-pol model, beginning with a discussion of the $3 \mathrm{~B}$ interaction. 


\section{A. Three-body Interaction}

Our development exploits the fact that the 3B interaction in water arises primarily from induction, with all other contributions vanishing quickly as the intermolecular separation increases 68170 This naturally leads to the following ansatz:

$$
V_{\mathrm{HBB} 2-\mathrm{pol}}^{3 B}=s_{3} V_{\text {poly }}^{3 B}+V_{\text {ind }}^{3 B},
$$

that represents the $3 \mathrm{~B}$ interaction as the sum of an induction term, $V_{i n d}^{3 B}$, and a short-range "correction", $V_{\text {poly }}^{3 B}$. The physical origins of $V_{\text {poly }}^{3 B}$ are related to the breakdown of the assumptions made in the derivation the Thole-type induction term as well as to the quantum-mechanical contributions associated with 3B exchange-repulsion and charge transfer 68 170/71 The induction scheme of TTM4-F is used in $V_{i n d}^{3 B}$ due to its superior accuracy with respect to other polarizable models (see Fig. 2). The short-ranged nature of the "correction" is enforced explicitly by the switching function, $s_{3}$,

$$
s_{3}=f\left(\xi_{12}\right) f\left(\xi_{13}\right)+f\left(\xi_{12}\right) f\left(\xi_{23}\right)+f\left(\xi_{13}\right) f\left(\xi_{23}\right),
$$

where

$$
f(\xi)= \begin{cases}1 & \xi \leq 0 \\ 1-3 \xi^{2}+2 \xi^{3} & 0<\xi \leq 1 \\ 0 & 1<\xi\end{cases}
$$

$\xi_{i j}=\left(R_{i j}-R_{I}\right) /\left(R_{F}-R_{I}\right), R_{i j}=\left|\boldsymbol{r}_{i}^{\mathrm{O}}-\boldsymbol{r}_{j}^{\mathrm{O}}\right|$, and $\boldsymbol{r}_{n}^{\mathrm{O}}$ denotes the position of the $n$-th molecule oxygen atom.
This form was found to be better capable of including all trimers in the first solvation shell of a central water molecule (in particular, the "linear" trimers) than those based on maximum oxygen-oxygen separations. Importantly, while the switch in Equation (3) goes from 3 to 0 as the trimer passes from the short-range to the long range, the product $s_{3} V_{\text {poly }}^{3 B}$ is fitted, rather than $V_{\text {poly }}^{3 B}$ by itself. This ensures that no artifact is introduced due to the switching. However, because $s_{3} V_{\text {poly }}^{3 B}$ is fitted in the context of $V_{\text {ind }}^{3 B}$, this also implies that, unlike in the case of the WHBB $3 B$ polynomial, $V_{\text {poly }}^{3 B}$ of HBB2-pol has no meaning by itself but only as the sum $s_{3} V_{\text {poly }}^{3 B}+V_{\text {ind }}^{3 B}$.

The ability of the short-range polynomial, $V_{\text {poly }}^{3 B}$, to accurately fit reference data depends largely on its degree, which also determines the associated numerical cost. Consequently, the large 5th and 6th degree $3 \mathrm{~B}$ polynomials in $V_{\text {poly }}^{3 B}$ of WHBB constitute the most computationally taxing part of the model. The different representation of the $3 \mathrm{~B}$ interactions (see Eq. (2)), along with the improved description of the induction energies in HBB2-pol allows for an accurate fit of the $\operatorname{CCSD}(\mathrm{T})$ reference data with a lower-degree polynomial. Specifically, in HBB2-pol the $V_{\text {poly }}^{3 B}$ part is a sum of second and third degree symmetrized products of exponentials of the intermolecular separations

$$
\eta_{i j}=\exp \left(-k\left|\boldsymbol{r}_{i}-\boldsymbol{r}_{j}\right|\right) \text {. }
$$

where $k$ is an adjustable parameter. Neither intramolecular distances nor the two-body terms - those which do not depend on the positions of all three molecules simultaneously - were included into the $V_{\text {poly }}^{3 B}$ in HBB2-pol. Labeling the three molecules as a, b, and c, there are 27 distances that contribute:

$$
\begin{aligned}
& \eta_{1}=\mathrm{e}^{-k_{\mathrm{HH}} d(\mathrm{Ha} 1, \mathrm{Hb} 1)}, \eta_{2}=\mathrm{e}^{-k_{\mathrm{HH}} d(\mathrm{Ha} 1, \mathrm{Hb} 2)}, \eta_{3}=\mathrm{e}^{-k_{\mathrm{HH}} d(\mathrm{Ha} 1, \mathrm{Hc} 1)}, \\
& \eta_{4}=\mathrm{e}^{-k_{\mathrm{HH}} d(\mathrm{Ha} 1, \mathrm{Hc} 2)}, \eta_{5}=\mathrm{e}^{-k_{\mathrm{HH}} d(\mathrm{Ha} 2, \mathrm{Hb} 1)}, \eta_{6}=\mathrm{e}^{-k_{\mathrm{HH}} d(\mathrm{Ha} 2, \mathrm{Hb} 2)} \text {, } \\
& \eta_{7}=\mathrm{e}^{-k_{\mathrm{HH}} d(\mathrm{Ha} 2, \mathrm{Hc} 1)}, \eta_{8}=\mathrm{e}^{-k_{\mathrm{HH}} d(\mathrm{Ha} 2, \mathrm{Hc} 2)}, \eta_{9}=\mathrm{e}^{-k_{\mathrm{HH}} d(\mathrm{Hb} 1, \mathrm{Hc} 1)}, \\
& \eta_{10}=\mathrm{e}^{-k_{\mathrm{HH}} d(\mathrm{Hb} 1, \mathrm{Hc} 2)}, \eta_{11}=\mathrm{e}^{-k_{\mathrm{HH}} d(\mathrm{Hb} 2, \mathrm{Hc} 1)}, \eta_{12}=\mathrm{e}^{-k_{\mathrm{HH}} d(\mathrm{Hb} 2, \mathrm{Hc} 2)} \text {, } \\
& \eta_{13}=\mathrm{e}^{-k_{\mathrm{OH}} d(\mathrm{Oa}, \mathrm{Hb} 1)}, \eta_{14}=\mathrm{e}^{-k_{\mathrm{OH}} d(\mathrm{Oa}, \mathrm{Hb} 2)}, \eta_{15}=\mathrm{e}^{-k_{\mathrm{OH}} d(\mathrm{Oa}, \mathrm{Hc} 1)} \text {, } \\
& \eta_{16}=\mathrm{e}^{-k_{\mathrm{OH}} d(\mathrm{Oa}, \mathrm{Hc} 2)}, \eta_{17}=\mathrm{e}^{-k_{\mathrm{OH}} d(\mathrm{Ob}, \mathrm{Ha} 1)}, \eta_{18}=\mathrm{e}^{-k_{\mathrm{OH}} d(\mathrm{Ob}, \mathrm{Ha} 2)}, \\
& \eta_{19}=\mathrm{e}^{-k_{\mathrm{OH}} d(\mathrm{Ob}, \mathrm{Hc} 1)}, \eta_{20}=\mathrm{e}^{-k_{\mathrm{OH}} d(\mathrm{Ob}, \mathrm{Hc} 2)}, \eta_{21}=\mathrm{e}^{-k_{\mathrm{OH}} d(\mathrm{Oc}, \mathrm{Ha} 1)} \text {, } \\
& \eta_{22}=\mathrm{e}^{-k_{\mathrm{OH}} d(\mathrm{Oc}, \mathrm{Ha} 2)}, \eta_{23}=\mathrm{e}^{-k_{\mathrm{OH}} d(\mathrm{Oc}, \mathrm{Hb} 1)}, \eta_{24}=\mathrm{e}^{-k_{\mathrm{OH}} d(\mathrm{Oc}, \mathrm{Hb} 2)} \text {, } \\
& \eta_{25}=\mathrm{e}^{-k_{\mathrm{OO}} d(\mathrm{Oa}, \mathrm{Ob})}, \eta_{26}=\mathrm{e}^{-k_{\mathrm{OO}} d(\mathrm{Oa}, \mathrm{Oc})}, \eta_{27}=\mathrm{e}^{-k_{\mathrm{OO}} d(\mathrm{Ob}, \mathrm{Oc})} \text {, }
\end{aligned}
$$

where $d(\mathrm{X}, \mathrm{Y})$ stands for the distance between atoms $\mathrm{X}$ and $Y$ (see also Eq.(5)). The monomials were constructed by symmetrizing the products of the $\eta_{n}$ variables with respect to the permutations of both the molecules and the hydrogen atoms within each molecule (48 elements in the permutation group total). A total of 131 different monomials were identified (13 are of second degree, and 
the remaining 118 are of third degree):

$$
\begin{aligned}
\kappa_{1} & =\eta_{5} \eta_{7}+\eta_{7} \eta_{9}+\eta_{10} \eta_{4}+\eta_{5} \eta_{9}+\eta_{6} \eta_{7}+\eta_{11} \eta_{2} \\
& +\eta_{2} \eta_{4}+\eta_{6} \eta_{8}+\eta_{2} \eta_{3}+\eta_{12} \eta_{4}+\eta_{11} \eta_{7}+\eta_{3} \eta_{9} \\
& +\eta_{11} \eta_{3}+\eta_{1} \eta_{3}+\eta_{5} \eta_{8}+\eta_{12} \eta_{2}+\eta_{10} \eta_{5}+\eta_{1} \eta_{4} \\
& +\eta_{1} \eta_{9}+\eta_{12} \eta_{8}+\eta_{11} \eta_{6}+\eta_{12} \eta_{6}+\eta_{10} \eta_{1}+\eta_{10} \eta_{8} \\
& \ldots \\
\kappa_{48} & =\eta_{19} \eta_{24} \eta_{6}+\eta_{10} \eta_{13} \eta_{18}+\eta_{15} \eta_{22} \eta_{9}+\eta_{10} \eta_{16} \eta_{22} \\
& +\eta_{19} \eta_{23} \eta_{7}+\eta_{13} \eta_{18} \eta_{7}+\eta_{20} \eta_{23} \eta_{4}+\eta_{19} \eta_{23} \eta_{3} \\
& +\eta_{11} \eta_{14} \eta_{18}+\eta_{19} \eta_{1} \eta_{23}+\eta_{12} \eta_{14} \eta_{18}+\eta_{14} \eta_{17} \eta_{4} \\
& +\eta_{10} \eta_{16} \eta_{21}+\eta_{16} \eta_{21} \eta_{2}+\eta_{19} \eta_{23} \eta_{5}+\eta_{11} \eta_{14} \eta_{17} \\
& +\eta_{13} \eta_{18} \eta_{8}+\eta_{12} \eta_{16} \eta_{22}+\eta_{11} \eta_{15} \eta_{22}+\eta_{12} \eta_{14} \eta_{17} \\
& +\eta_{15} \eta_{1} \eta_{21}+\eta_{14} \eta_{18} \eta_{7}+\eta_{20} \eta_{23} \eta_{8}+\eta_{13} \eta_{17} \eta_{9} \\
& +\eta_{15} \eta_{21} \eta_{2}+\eta_{13} \eta_{17} \eta_{4}+\eta_{20} \eta_{24} \eta_{4}+\eta_{14} \eta_{18} \eta_{8} \\
& +\eta_{19} \eta_{24} \eta_{7}+\eta_{20} \eta_{23} \eta_{5}+\eta_{12} \eta_{16} \eta_{21}+\eta_{19} \eta_{24} \eta_{2} \\
& +\eta_{13} \eta_{17} \eta_{3}+\eta_{20} \eta_{24} \eta_{2}+\eta_{16} \eta_{22} \eta_{5}+\eta_{1} \eta_{20} \eta_{23} \\
& +\eta_{15} \eta_{22} \eta_{6}+\eta_{14} \eta_{17} \eta_{3}+\eta_{20} \eta_{24} \eta_{6}+\eta_{15} \eta_{21} \eta_{9} \\
& +\eta_{10} \eta_{13} \eta_{17}+\eta_{13} \eta_{18} \eta_{9}+\eta_{15} \eta_{22} \eta_{5}+\eta_{11} \eta_{15} \eta_{21} \\
& +\eta_{16} \eta_{22} \eta_{6}+\eta_{16} \eta_{1} \eta_{21}+\eta_{19} \eta_{24} \eta_{3}+\eta_{20} \eta_{24} \eta_{8} \\
& \ldots \\
\kappa_{131} & =\eta_{25} \eta_{26} \eta_{27} .
\end{aligned}
$$

The $V_{\text {poly }}^{3 B}$ itself was then taken as a linear combination of $\kappa_{n}$ :

$$
V_{\text {poly }}^{3 B}=\sum_{n=1}^{131} v_{n} \kappa_{n},
$$

with the coefficients $v_{n}$ obtained using the least squares fit ${ }^{72}$ to the $\operatorname{CCSD}(\mathrm{T})$ data. The gradient of $V_{\text {poly }}^{3 B}$ with respect to the atomic positions was computed using MAPLE $[73$

\section{B. Composition of three-body training set}

After translational and rotational invariance, the $3 \mathrm{~B}$ interaction potential for flexible water molecules is $21 \mathrm{di}-$ mensional. Since this high-dimensional PES cannot be readily trained on a grid, a training set representative of the "important regions" of the 3B PES was generated by including: 1) repulsive configurations with positive binding energies that are compressed relative to the trimer global minimum, 2) "low-energy" configurations with thermally accessible binding energies, and 3) long-range trimer configurations that have weak $3 \mathrm{~B}$ interactions. The total training set consists of 8019 trimer configurations for which the $3 \mathrm{~B}$ energies were computed at the $\operatorname{CCSD}(\mathrm{T}) /$ aug-cc-pVTZ level and corrected for BSSE $[56$

The majority of the configurations in the training set correspond to an expanded set of "low-energy" configurations, similar in composition to that used in the analysis

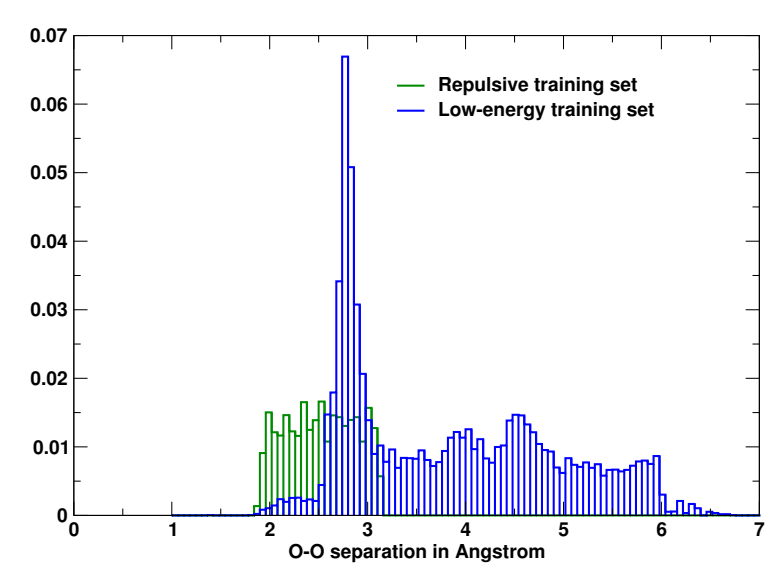

FIG. 3. Distribution of O-O distances in the three-body training set, including all three $\mathrm{O}-\mathrm{O}$ distances per trimer. "Lowenergy" denotes configurations which were thermally accessible (see text for details).

of section II]. Of the 5515 thermally accessible configurations that were used in the fit, 996 trimers were selected from MD simulations of clusters (trimers and hexamers) at $30 \mathrm{~K}$ on the WHBB potential energy surface, 3311 trimers were extracted from classical MD simulations of hexagonal ice and liquid water carried out with TTM3-F at $50 \mathrm{~K}$ and $300 \mathrm{~K}$ respectively, 792 trimers were obtained by randomly orienting water monomers in geometries near the global minimum, and 416 trimers were obtained from scans of low-energy structures.

The long-range portion of the training set consists of 432 weakly interacting trimers, with an average O-O distance of at least $5.5 \AA$ between monomers. After verifying that the $\operatorname{CCSD}(\mathrm{T})$ /aug-cc-pVTZ 3B interaction energy for these long-range configurations was in agreement with the 3B induction energy from TTM4-F (within the error associated with basis-set truncation), we assigned these configurations the TTM4-F 3B induction energy. This enforces the "boundary condition" that the 3B interaction of HBB2-pol become pure induction at long distances.

It is important to mention that our initial model were fitted to a training set that emphasized only the lowenergy and long-range regions, which is consistent with the parameterization strategy adopted for the WHBB $3 \mathrm{~B}$ potential ${ }^{51}$. While the resulting models succeeded in predicting the relative stabilities of trimer and hexamer isomers, we found that they were numerically unstable in MD simulations of larger clusters, such as the 32-mer. This was related to insufficient coverage of the repulsive, short-ranged region of the trimer PES. To address this instability, 2072 configurations were generated by performing random rotations on monomers that were compressed relative to the trimer minimum geometry. As shown in Figure 3 this repulsive training set evenly covers O$\mathrm{O}$ distances from $1.8 \AA$ to $3.2 \AA$. Many of these compressed configurations, however, correspond to trimers that would practically never be sampled in simulations 


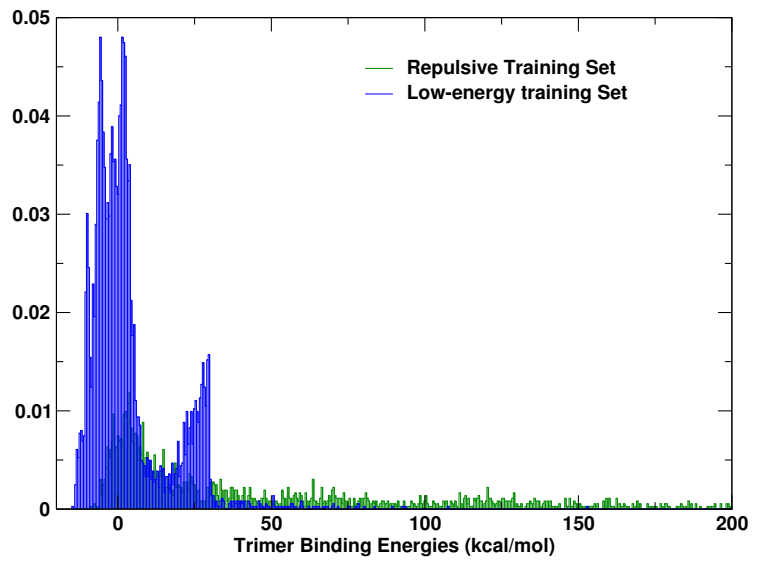

FIG. 4. Distribution of trimer binding energies in the threebody training set.

of water under ambient conditions. While including these configurations in the training set was required to ensure the numerical stability of the model, it was also necessary to guarantee that these highly-repulsive configurations did not degrade the quality of the fit in the region near the minimum. This consideration is discussed in the following section.

\section{Testing the accuracy of the three-body fit}

In order to assess the accuracy of any model with respect to $a b$ initio data, it would be ideal to parameterize the model based on one set of reference data (the "training" set) and validate the model using a separate set of data (the "testing" set). However, due to the large computational cost associated with BSSEcorrected CCSD(T)/aug-cc-pVTZ 3B interactions and the fact that the model should be parametrized to as large of a reference set as possible, having two distinct sets is unfeasible. In order to estimate the bias associated with training and testing on the same reference data, the complete training set was equally divided into two parts, a training set and a testing set. Care was taken to preserve the relative compositions of each set, e.g., half of the 972 configurations extracted from simulations of ice were randomly selected and placed in the training set while the other half was placed in the testing set.

Since the goal of HBB2-pol is to describe water from small molecular clusters in the gas phase to condensed phases, we found it important to decompose the RMS into categories: 1) trimers with a binding energy of less than $5 \mathrm{kcal} / \mathrm{mol}$, which are particularly important for low-temperature cluster isomer relative stabilities, 2) trimers with a binding energy of less than $30 \mathrm{kcal} / \mathrm{mol}$, which are sampled during path-integral molecular dynamics (PIMD) simulations of bulk water at ambient conditions, and 3) the complete training set, regardless of binding energy (see Table II). Due to the proximity of the
TABLE I. RMS deviation of three-body interactions from trimers with binding energies less than $5 \mathrm{kcal} / \mathrm{mol}$, less than $30 \mathrm{kcal} / \mathrm{mol}$, and for the complete training set. See main text for details.

\begin{tabular}{rccc}
\hline & WHBB & $V_{\text {ind }}^{3 B}$ & HBB2-pol \\
\cline { 2 - 4 } & \multicolumn{3}{c}{ RMS for Training Set } \\
\hline $\mathrm{E}_{\text {bind }}<5$ & 0.10 & 0.13 & 0.06 \\
$\mathrm{E}_{\text {bind }}<30$ & 0.14 & 0.35 & 0.10 \\
Total & 0.69 & 2.26 & 0.85 \\
\hline \multicolumn{4}{c}{ RMS for Testing Set } \\
\hline $\mathrm{E}_{\text {bind }}<5$ & 0.10 & 0.13 & 0.06 \\
$\mathrm{E}_{\text {bind }}<30$ & 0.15 & 0.35 & 0.16 \\
Total & 0.68 & 2.20 & 0.82 \\
\hline \multicolumn{4}{c}{ RMS for Complete Set } \\
\hline $\mathrm{E}_{\text {bind }}<5$ & 0.10 & 0.13 & 0.05 \\
$\mathrm{E}_{\text {bind }}<30$ & 0.14 & 0.34 & 0.11 \\
Total & 0.66 & 2.17 & 0.83 \\
\hline
\end{tabular}

energies for different isomers of the small clusters, it is desirable that $3 \mathrm{~B}$ interactions corresponding to clusters with the lowest binding energies have the lowest RMS. As was discussed in Section III B, it was necessary to include $3 \mathrm{~B}$ energies corresponding to trimers with positive binding energies to ensure the stability of HBB2-pol. At the same time, care was taken to ensure that the accuracy in the low-energy region was not diluted by being needlessly accurate for trimers with enormous binding energies that will rarely be sampled. By appropriately weighting the reference data, HBB2-pol achieves the best accuracy in the low-energy region, slightly larger RMS in the intermediate region $\left(E_{\text {bind }}<5 \mathrm{kcal} / \mathrm{mol}\right)$, and the largest error for those configurations with binding energies greater than $30 \mathrm{kcal} / \mathrm{mol}$. To obtain this RMS distribution, configurations with $3 \mathrm{~B}$ interactions were weighted according to their trimer binding energies, where configurations with $E_{\text {bind }}<15 \mathrm{kcal} / \mathrm{mol}$ were given a weight of 1.0 , while weights for configurations binding energies larger than $15 \mathrm{kcal} / \mathrm{mol}$ a weight of $e^{-a\left(E_{b i n d}-E_{0}\right)}$, where $\mathrm{a}=$ $0.05 \mathrm{kcal} / \mathrm{mol}^{-1}$ and $E_{0}$ was $15 \mathrm{kcal} / \mathrm{mol}$.

The results presented in Table $\mathrm{I}$ confirms the ability of the HBB2-pol 3B function to recover the ab initio data and demonstrates that the training set is sufficiently large to render the model insensitive to the size of the training set. This analysis does not, however, probe whether the training set includes all the physically relevant configurations. Assessing whether the composition of the training set is biased can only be accomplished by examining $a b$ initio properties such as relative cluster isomer stabilities, experimental properties such as virial coefficients, and the overall numerical stability of the model. 


\section{HBB2-pol}

Using the 3B interaction proposed in Eq. (2), HBB2pol has been developed through the many-body expansion, Eq. (1). The spectroscopically-accurate monomer potential energy surface of Partridge and Schwenke is used for the $1 \mathrm{~B}$ terms ${ }^{[74}$ For the $2 \mathrm{~B}$ interaction, the HBB2 PES ${ }^{69}$ is employed at short-range. The HBB2 short-range $2 \mathrm{~B}$ interaction is smoothly switched to electrostatics/induction plus dispersion term at long-range over the interval $R_{I}^{2 B}=5.5 \AA<R_{\mathrm{OO}}<R_{F}^{2 B}=7.5 \AA$ :

$$
\begin{aligned}
V^{2 B} & =\left(1-s_{2}\right) V_{H B B 2}^{2 B} \\
& +s_{2}\left[V_{\text {elec }}^{2 B}+V_{\text {ind }}^{2 B}-\frac{C_{6}}{R_{\mathrm{OO}}^{6}}\right], \\
s_{2} & = \begin{cases}0 & \xi \leq 0 \\
\xi^{3}\left(10-15 \xi+6 \xi^{2}\right) & 0<\xi \leq 1, \\
1 & 1<\xi\end{cases}
\end{aligned}
$$

where $\xi=\left(R_{\mathrm{OO}}-R_{I}\right) /\left(R_{F}-R_{I}\right)$. The $V_{\text {elec }}^{2 B}$ and $V_{\text {ind }}^{2 B}$ terms have the same form as TTM4-F, and the value of $C_{6}$ was taken as the difference between the sum of the $a b$ initio van der Waals constants describing the dispersion and induction interactions in the asymptotic region from Ref. 49, and the $1 / R_{\mathrm{OO}}^{6}$ coefficient of the isotropic part of $V_{\text {ind }}^{2 B}$

$$
C_{6}=(47.053232 \text { a.u. }+10.66517 \text { a.u })-2 \alpha \mu^{2},
$$

(using the isotropic molecular polarizability given by TTM4-F, $\alpha=1.41567 \AA^{3}$ the molecular dipole $\mu=$ $1.864047 \mathrm{D})$. The 3B interactions are those presented in Eq. (2), and all the higher-body terms are approximated by the induction energy as in TTM4-F,

$$
\begin{aligned}
V^{N B} & =V_{i n d}^{N B}-\sum_{i<j<k}^{N} V_{i n d}^{3 B}(i, j, k) \\
& -\sum_{i<j}^{N} V_{i n d}^{2 B}(i, j) .
\end{aligned}
$$

Since $V_{H B B 2}^{2 B}$ accounts for polarization at the $2 \mathrm{~B}$ level, the short-range $2 \mathrm{~B}$ contribution must be subtracted from the N-body induction to prevent double counting. This problem does not arise for the 3B interactions since induction is not modified at the 3B level (Eq. (2)). The HBB2-pol interaction energy for $N$ water molecules is thus given by the following expression

$$
\begin{aligned}
& E_{N-\text { mer }}=\sum_{i}^{N} V_{P S}^{1 B}(i) \\
& +\sum_{i<j}^{N}\left\{\left(1-s_{2}\right)\left[V_{H B B 2}^{2 B}-V_{\text {ind }}^{2 B}\right]+s_{2}\left[V_{\text {elec }}^{2 B}-\frac{C_{6}}{R_{\mathrm{OO}}^{6}}\right]\right\} \\
& +\sum_{i<j<k}^{N} s_{3} V_{\text {poly }}^{3 B}(i, j, k)+V_{\text {ind }}^{N B}(1, \ldots, N)
\end{aligned}
$$

where the $3 \mathrm{~B}$ switching functions, $s_{3}$, is given by Eq. (3).

\section{RESULTS}

In this section we demonstrate the ability HBB2-pol to reproduce $\operatorname{CCSD}(\mathrm{T})$ calculations and the experimental second and third virial coefficients.

\section{A. Short-range Three-body Interaction Addresses Systematic Flaws in Polarizable Models}

As discussed above, the three-body interactions primarily originate from induction, though for more strongly bound clusters effects including exhangerepulsion and charge transfer can also make a significant contribution. 6870 As a consequence, force fields which treat only induction are inherently unable to fully describe 3B interactions. By contrast, models that only treat short-range $3 \mathrm{~B}$ interactions are unable to describe the induction interactions that dominate at long range. This is illustrated in Figure 5, where HBB2-pol is compared with WHBB, TTM4-F 3B induction, and CCSD(T)/aug-cc-pVTZ data along two representative cuts through the water trimer PES. The CCSD(T) reference data used for this comparison were not included in the training set. This comparison clearly shows that the addition of the short-range "correction" to the induction brings the 3B interactions of HBB2-pol into close agreement with the $\operatorname{CCSD}(\mathrm{T})$ data.

\section{B. Trimer stationary points}

To assess the combined accuracy of the 1B, 2B, and 3B interactions of HBB2-pol, we studied the relative energies of four water trimer isomers identified in Table II by their free-hydrogen orientation: "u" for pointing up, "p" if the hydrogen lies in the plane of the oxygen atoms, and " $\mathrm{d}$ " for pointing down. The energetics of these structures have been reported in Ref. 75, where geometries optimized at the MP2/aug-cc-pVQZ level were used to calculate the energies at the $\operatorname{CCSD}(\mathrm{T})$ level in the complete basis limit. The HBB2-pol energies relative to the trimer global minimum (uud) are reported in Table II for the geometries optimized on the HBB2-pol PES. These favorably interacting trimers have $\operatorname{CCSD}(\mathrm{T})$ binding energies of approximately $-15 \mathrm{kcal} / \mathrm{mol}$, which implies that the energies separating these stationary points are on the order of $1-10 \%$ of the binding energy. The HBB2-pol relative energies fall within $0.05 \mathrm{kcal} / \mathrm{mol}$ of the reference data for the upd and uuu structures, while a larger difference of $0.23 \mathrm{kcal} / \mathrm{mol}$ is obtained for the ppp structure. HBB2-pol, however, is not expected to achieve perfect agreement with the $\operatorname{CCSD}(\mathrm{T}) / \mathrm{CBS}$ data ${ }^{75}$ due to the different basis set used in the fit of the $3 \mathrm{~B}$ terms. 


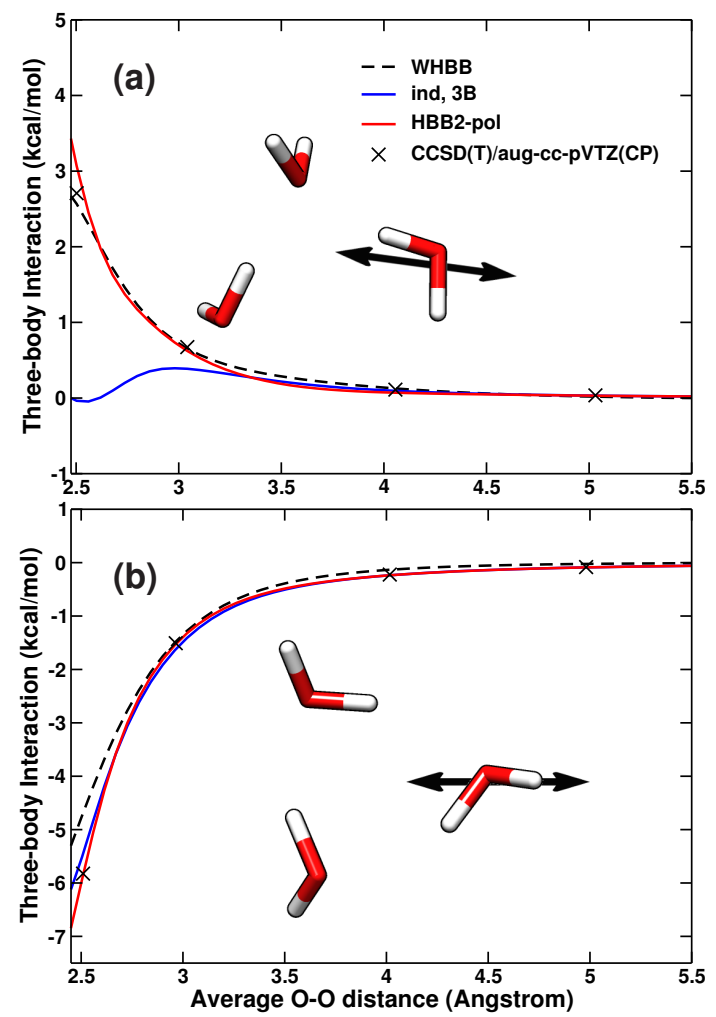

FIG. 5. Three-body interaction energy for two cuts through the water trimer potential energy surface: WHBB (black dashes), three-body induction (blue), HBB2-pol (red) and $\operatorname{CCSD}(\mathrm{T}) /$ aug-cc-pVTZ (crosses).

TABLE II. Relative energies of water trimer isomers with respect to the global minimum "uud" in kcal/mol. $\operatorname{CCSD}(\mathrm{T})$ energies were extrapolated to complete basis set limit, from Ref. 75 .

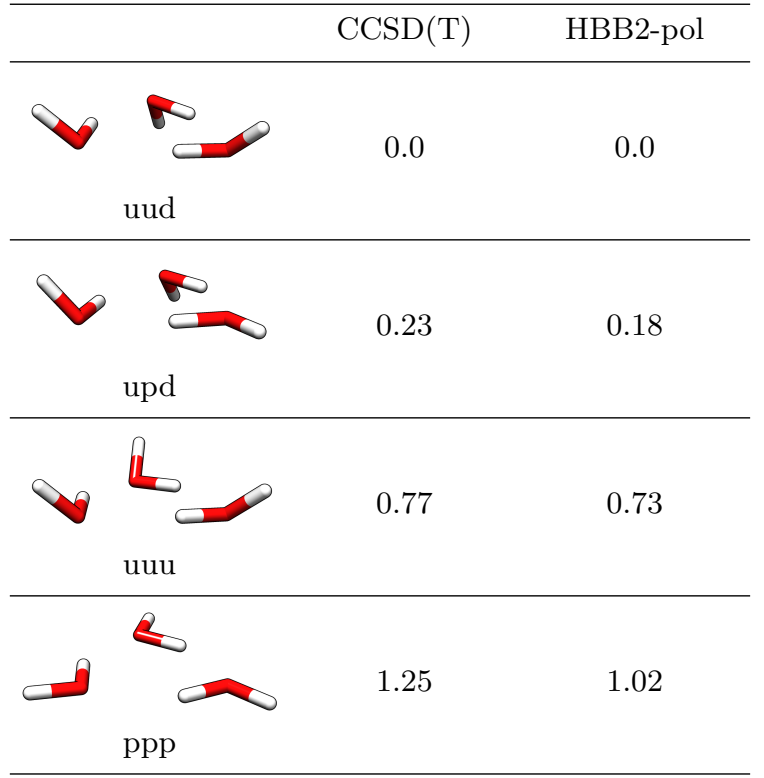

\section{Virial coefficients}

Virial coefficients are derived from the virial equation of state that expresses $p / k_{B} T$ as a power series in density and gauge deviations from the ideal gas behavior,

$$
\frac{p}{k_{B} T}=\frac{N}{V}\left[1+B_{2} \frac{N}{V}+B_{3}\left(\frac{N}{V}\right)^{2}+\ldots\right] .
$$

Here, $B_{2}$ and $B_{3}$ are the second and third virial coefficients, respectively ${ }^{76}, 78$ The second virial coefficient depends only on the pair interaction while the third virial coefficient also includes the $3 \mathrm{~B}$ interaction, but no $(n>3)$-body energies. Since both $B_{2}$ and $B_{3}$ are experimentally accessible, the virial coefficients provide a critical assessment of the accuracy of water potentials.

Neglecting the contribution of intramolecular vibrational modes (that is, assuming rigid monomers) and nuclear quantum effects, the second virial coefficient is given by ${ }^{78}$,

$$
\begin{aligned}
B_{2}(T) & =-2 \pi \int \mathrm{d} R_{12} R_{12}^{2}\left\langle f_{12}\right\rangle_{\boldsymbol{\Omega}_{1}, \boldsymbol{\Omega}_{2}}, \\
f_{12} & =\mathrm{e}^{-\beta V^{2 B}\left(R_{12}, \boldsymbol{\Omega}_{1}, \boldsymbol{\Omega}_{2}\right)}-1,
\end{aligned}
$$

where $\beta=1 / k_{B} T$ is the inverse temperature, $R_{12}$ is the distance between the monomer centers of mass, $V^{2 B}\left(R_{12}, \boldsymbol{\Omega}_{\mathbf{1}}, \boldsymbol{\Omega}_{\mathbf{2}}\right)$ is the intermolecular interaction energy, and the angular brackets stand for the average over the orientations of the molecules $\boldsymbol{\Omega}_{1,2}$. The Mayer function, $f_{12}$, has the useful property of going to zero as the molecules move apart. To numerically evaluate Eq. 10, the Simpson rule is used to calculate the radial component of the integral, while Monte Carlo integration is used to evaluate the orientational average using $10^{5}$ random orientations of the monomers at each point on the radial grid.

To explore the sensitivity of the second virial coefficient to the choice of the rigid monomer geometry, its values are calculated using two different configurations: the Born-Oppenheimer minimum energy configuration given by the Partridge-Schwenke potential energy surface $\left(r_{O H}^{e q}=0.95784 \AA \text { and } \theta_{H O H}^{e q}=104.508^{\circ}\right)^{74}$, and the ground-state vibrationally-averaged configuration of $r_{O H}^{e q}=0.9716256 \AA$ and reported in reference 80 $\left(\theta_{\mathrm{HOH}}^{e q}=104.69^{\circ}\right)$. Importantly, examining the results for these two configurations provides not only an estimate of the effect of flexibility, but also of nuclear quantum effects "sensed" through the ground-state vibrationally averaged configuration.

Plotted in Figure 6 is the difference between the calculated virial coefficients and the experimental data! 79 Since $B_{2}$ is the integral of $e^{-\beta V^{2 B}}-1$, comparison to lowtemperature results are particularly interesting as these are most sensitive to the region near the dimer minimum geometry. For the Born-Oppenheimer equilibrium geometries (Fig. 6a), the $a b$ initio-based potential energy surfaces WHBB and HBB2-pol very closely reproduce the experimental data. Since HBB2-pol and WHBB 
FIG. 6. Effect of the rigid monomer configuration on the error in the classical second virial coefficient relative to experiment ${ }^{[9}$ Differences between WHBB and HBB2-pol were indistinguishable on the scale of this plot, so both have been assigned to the red line. For E3B, its rigid monomer geometry was used in both plots.

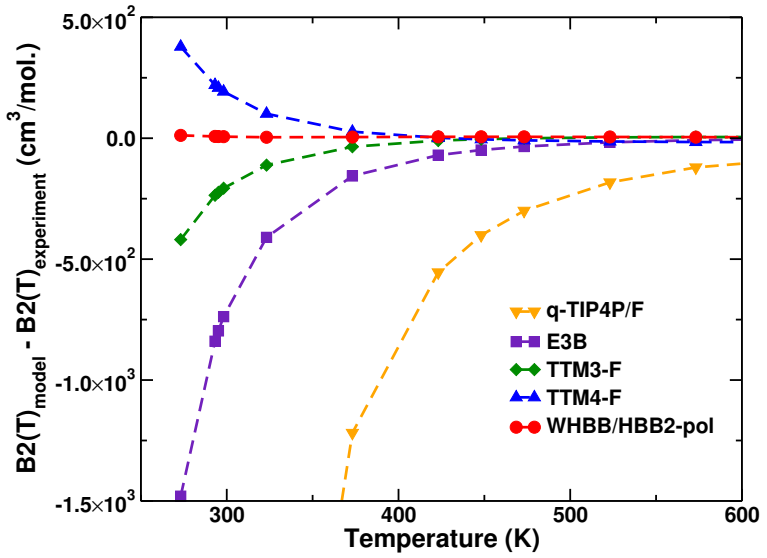

(a) Equilibrium monomer geometry

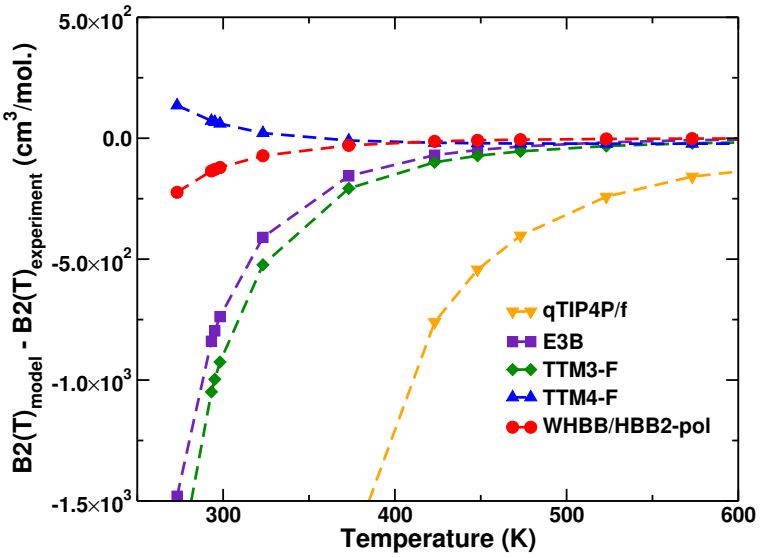

(b) Vibrationally-averaged monomer geometry

crease in the value of $B_{2}(T)$ for most models. TTM3$\mathrm{F}$, however, exhibits a large change in its second virial coefficient. This is likely due to the empirical modification of the dipole moment surface, which only affects TTM3-F when the monomer distorts from the equilibrium configuration.29

The third virial coefficient depends on the interaction of trimers and provides an indirect measure of the $3 \mathrm{~B}$ interaction. Following Hill 176 , the third virial coefficient can be separated into a pairwise component, $B_{3}^{0}(T)$, and the $3 \mathrm{~B}$ contribution, $\Delta B_{3}^{3 B}(T)$ :

$$
B_{3}(T)=B_{3}^{0}(T)+\Delta B_{3}^{3 B}(T)
$$

where the pairwise contribution is the integral over the product of the three Mayer functions in Eq. (12), and the $3 \mathrm{~B}$ contribution is given by Eq. (13).

$$
\begin{gathered}
B_{3}^{0}(T)=-\frac{8}{3} \pi^{2} \int \mathrm{d} R_{12} \mathrm{~d} R_{13} R_{12}^{2} R_{13}^{2}\left\langle f_{12} f_{13} f_{23} \sin \vartheta_{(2,1,3)}\right\rangle_{\boldsymbol{\Omega}_{1}, \boldsymbol{\Omega}_{2}, \boldsymbol{\Omega}_{3}, \vartheta_{(2,1,3)}}, \\
\Delta B_{3}^{3 B}(T)=-\frac{8}{3} \pi^{2} \int \mathrm{d} R_{12} \mathrm{~d} R_{13} R_{12}^{2} R_{13}^{2}\left\langle\left[\mathrm{e}^{-\beta V_{1,2,3}^{3 B}}-1\right] \mathrm{e}^{-\beta\left(V_{1,2}^{2 B}+V_{1,3}^{2 B}+V_{2,3}^{2 B}\right)} \sin \vartheta_{(2,1,3)}\right\rangle_{\boldsymbol{\Omega}_{\mathbf{1}}, \boldsymbol{\Omega}_{\mathbf{2}}, \boldsymbol{\Omega}_{\mathbf{3}}, \vartheta(2,1,3)} .
\end{gathered} .
$$

Following the work of Tainter et al.,24 we computed the third virial coefficient by fixing one molecule at the origin, evaluating two radial integrals through the twodimensional Simpson rule, and using Monte Carlo integration for the 9 orientational degrees of freedom and the angle $\vartheta_{(2,1,3)}$ between the centers of mass of molecules 2 , 1,3 at each radial grid point (using $10^{6}$ monomer orientations). This integration strategy was demonstrated in Ref. 24 to recover the results from the more efficient Mayer sampling approach. ${ }^{81}$ Our implementation reproduces the data from Ref. 24 for the E3B model.

Rigid, classical third virial coefficients using vibrationally-averaged monomer geometries are reported in figure 7. At lower temperatures, HBB2-pol agrees with WHBB. In the high temperature limit, however, HBB2-pol compare more favorably with 


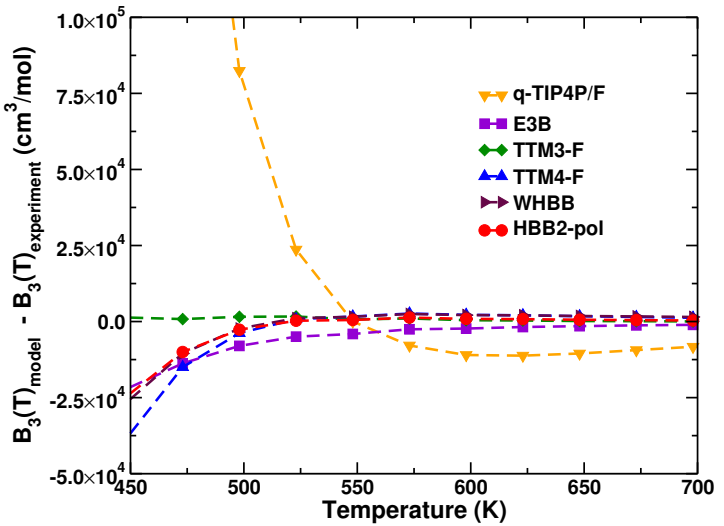

FIG. 7. Rigid, classical third virial coefficient using vibrationally-averaged monomer geometries. Experimental data from Ref. 82 .

experiment than WHBB, which is consistent with HBB2-pol providing a more accurate description of the $3 \mathrm{~B}$ interactions. The errors in the $2 \mathrm{~B}$ and $3 \mathrm{~B}$ interactions of TTM3-F appear to cancel one another, resulting in an third virial coefficient that is remarkably close to experiment. Much work has been invested in exploring the role of nuclear quantum effects $\$ 9|83| 84$ and monomer flexibility ${ }^{85}$ While the exploration of the monomer configuration on the second virial coefficient indicates that flexibility and nuclear quantum effects are important, these results are by no means conclusive. We will pursue a more rigorous characterization of the effect of flexibility and nuclear quantization in future work.

\section{SUMMARY}

In this study, the accuracy of several force fields, semiempirical methods, DFT, and $a b$ initio-based models in reproducing the two- and three-body water interactions was assessed against BSSE-corrected CCSD(T)/aug-cc-pVTZ data. Our analysis of the manybody expansion of the interaction energy indicates that defects inherent to polarizable models, which are nonnegligable when molecules are close to one another, can be effectively corrected through an explicit short-range term expressed in terms of permutationally invariant polynomials. Based on these findings, we developed a new water model, HBB2-pol, that is derived entirely from "first principles". HBB2-pol achieves excellent accuracy with respect to the $\operatorname{CCSD}(\mathrm{T})$ data for the twoand three-body interactions, isomer relative energies of small clusters, and second and third virial coefficients. Importantly, the inclusion of explicit polarization in the three-body interaction term enables the use of relatively low-degree polynomials, which, in turn, results in a significant decrease in the computational cost associated with HBB2-pol relative to other ab initio-based models.
Through its combined accuracy and computational efficiency, HBB2-pol thus opens the doorway to fully "first principles" simulations of water in the condensed phases, which will help resolve current controversies 86 .91.

\section{ACKNOWLEDGEMENT}

This research was supported by the National Science Foundation through grant CHE-1111364. We are grateful to the National Science Foundation for a generous allocation of computing time on Xsede resources (award TG-CHE110009). Additionally, we would like to thank Chris Mundy and Greg Schenter for their assistance in calculations involving SCP-NDDO.

${ }^{1}$ Dahlke, E.; Olson, R.; Leverentz, H.; Truhlar, D. J. Phys. Chem. A 2008, 112, 3976-84.

${ }^{2}$ Bates, D.; Tschumper, G. J. Phys. Chem. A 2009, 113, 35553559 .

${ }^{3}$ Góra, U.; Podeszwa, R.; Cencek, W.; Szalewicz, K. J. Chem. Phys. 2011, 135, 224102.

${ }^{4}$ Soper, A.; Benmore, C. Phys. Rev. Lett. 2008, 101, 065502.

${ }^{5}$ Paesani, F.; Voth, G. J. Phys. Chem. B 2009, 113, 5702-19.

${ }^{6}$ Wang, Y.; Babin, V.; Bowman, J.; Paesani, F. J. Am. Chem. Soc. 2012, 134, 11116-9.

${ }^{7}$ Markland, T.; Berne, B. Proc. Natl. Acad. Sci. 2012, 109, 79887991.

${ }^{8}$ Hankins, D.; Moskowitz, J.; Stillinger, F. J. Chem. Phys. 1970, $53,4544-4554$.

${ }^{9}$ Xantheas, S. J. Chem. Phys. 1994, 100, 7523-7534.

${ }^{10}$ Xantheas, S. Chem. Phys. 2000, 258, 225-231.

${ }^{11}$ Defusco, A.; Schofield, D.; Jordan, K. Mol. Phys. 2007, 105, 2681-2696.

${ }^{12}$ Kumar, R.; Wang, F.; Jenness, G.; Jordan, K. J. Chem. Phys. 2010, 132, 014309.

${ }^{13}$ Hodges, M.; Stone, A.; Xantheas, S. J. Phys. Chem. A 1997, 101, 9163-9168.

${ }^{14}$ Ojamie, L.; Hermansson, K. J. Phys. Chem. 1994, 98, 42714282.

${ }^{15}$ Pedulla, J.; Vila, F.; Jordan, K. J. Chem. Phys. 1996, 105, 11091.

${ }^{16}$ Cui, J.; Liu, H.; Jordan, K. J. Phys. Chem. B 2006, 110, 18872 18878.

${ }^{17}$ Hermann, A.; Krawczyk, R.; Lein, M.; Schwerdtfeger, P.; Hamilton, I.; Stewart, J. Phys. Rev. A 2007, 76, 013202.

${ }^{18}$ Jorgensen, W.; Chandrasekhar, J.; Madura, J.; Impey, R.; Klein, M. J. Chem. Phys. 1983, 79, 926.

${ }^{19}$ Berendsen, H.; Postma, J.; van Gunsteren, W.; Hermans, J. In Intermolecular Forces; Pullman, B., Ed.; Reidel Publishing Company: Dordrecht, 1981; pp 333-342.

${ }^{20}$ Habershon, S.; Markland, T.; Manolopoulos, D. J. Chem. Phys. 2009, 131, 024501.

${ }^{21}$ Paesani, F.; Zhang, W.; Case, D.; Cheatham, T.; Voth, G. J. Chem. Phys. 2006, 125, 184507.

${ }^{22}$ Vega, C.; Abascal, J. Phys. Chem. Chem. Phys. 2011, 13, 1966319688.

${ }^{23}$ Kumar, R.; Skinner, J. J. Phys. Chem. B 2008, 112, 8311-8318.

${ }^{24}$ Tainter, C.; Pieniazek, P.; Lin, Y.; Skinner, J. J. Chem. Phys. 2011, 134, 184501.

${ }^{25}$ Tainter, C.; Skinner, J. J. Chem. Phys. 2012, 137, 104304.

${ }^{26}$ Lopes, P.; Roux, B.; Mackerell, A. Theor. Chem. Acc. 2009, 124, 11-28.

${ }^{27}$ Applequist, J.; Carl, J.; Fung, K. J. Am. Chem. Soc. 1972, 94, 2952-2960.

${ }^{28}$ Thole, B. Chem. Phys. 1981, 59, 341-350.

${ }^{29}$ Fanourgakis, G.; Xantheas, S. J. Chem. Phys. 2008, $128,074506$. 
${ }^{30}$ Burnham, C.; Anick, D.; Mankoo, P.; Reiter, G. J. Chem. Phys. 2008, 128, 154519.

${ }^{31}$ Ren, P.; Ponder, J. J. Phys. Chem. B 2003, 107, 5933-5947.

${ }^{32}$ Stewart, J. J. Comput. Chem. 1989, 10, 209-220.

${ }^{33}$ Bernal-Uruchurtu, M.; Ruiz-López, M. Chem. Phys. Lett. 2000, 330, 118-124.

${ }^{34}$ Chang, D.; Schenter, G.; Garrett, B. J. Chem. Phys. 2008, 128, 164111.

${ }^{35}$ Murdachaew, G.; Mundy, C.; Schenter, G.; Laino, T.; Hutter, J. J. Phys. Chem. A 2011, 115, 6046-53.

${ }^{36}$ Becke, A. Phys. Rev. A 1988, 38, 3098-3100.

${ }^{37}$ Lee, C.; Yang, W.; Parr, R. Phys. Rev. B 1988, 37, 785-789.

${ }^{38}$ Perdew, J.; Burke, K.; Ernzerhof, M. Phys. Rev. Lett. 1996, 77, 3865-3868

${ }^{39}$ Grimme, S. J. Comput. Chem. 2004, 25, 1463-1473.

${ }^{40}$ Grimme, S. J. Comput. Chem. 2006, 27, 1787-1799.

${ }^{41}$ Fulton, J.; Schenter, G.; Baer, M.; Mundy, C.; Dang, L.; Balasubramanian, M. J. Phys. Chem. B 2010, 114, 12926-12937.

${ }^{42}$ Ma, Z.; Zhang, Y.; Tuckerman, M. J. Chem. Phys. 2012, 044506 , 044506 .

${ }^{43}$ Grimme, S.; Antony, J.; Ehrlich, S.; Krieg, H. J. Chem. Phys. 2010, 132, 154104.

${ }^{44}$ Dion, M.; Rydberg, H.; Schröder, E.; Langreth, D.; Lundqvist, B. Phys. Rev. Lett 2004, 92, 246401.

${ }^{45}$ Lee, K.; Murray, E.; Kong, L.; Lundqvist, B.; Langreth, D. Phys. Rev. B 2010, 82, 081101.

${ }^{46}$ Vydrov, O.; Van Voorhis, T. J. Chem. Phys. 2010, 133, 244103.

${ }^{47}$ Wang, J.; Román-Pérez, G.; Soler, J.; Artacho, E.; FernándezSerra, M. J. Chem. Phys. 2011, 134, 024516.

${ }^{48}$ Murray, E.; Galli, G. Phys. Rev. Lett. 2012, 108, 105502.

${ }^{49}$ Bukowski, R.; Szalewicz, K.; Groenenboom, G.; van Der Avoird, A. J. Chem. Phys. 2008, 128, 094314.

${ }^{50}$ Bukowski, R.; Szalewicz, K.; Groenenboom, G.; van Der Avoird, A. J. Chem. Phys. 2008, 128, 094313.

${ }^{51}$ Wang, Y.; Huang, X.; Shepler, B.; Braams, B.; Bowman, J. J. Chem. Phys. 2011, 134, 094509

${ }^{52}$ Bukowski, R.; Szalewicz, K.; Groenenboom, G.; van der Avoird, A. Science 2007, 315, 1249-52.

${ }^{53}$ Leforestier, C.; Szalewicz, K.; van der Avoird, A. J. Chem. Phys. 2012, 137, 014305.

${ }^{54}$ Raghavachari, K.; Truck, G.; Pople, J.; Head-Gordon, M. Chem. Phys. Lett. 1989, 157, 479-483.

${ }^{55}$ Dunning, T. J. Chem. Phys. 1989, 90, 1007.

${ }^{56}$ Boys, S.; Bernardi, F. Mol. Phys. 1970, 19, 553.

${ }^{57}$ Weigend, F.; Ahlrichs, R. Phys. Chem. Chem. Phys. 2005, 7, 3297-3305

${ }^{58}$ Schafer, A.; Huber, C.; Ahlrichs, R. J. Chem. Phys. 1994, 100, $5829-5835$

${ }^{59}$ Wennmohs, F.; Neese, F. Chem. Phys. 2008, 343, 217-230.

${ }^{60}$ Walker, R. C.; Crowley, M. F.; Case, D. A. J. Comput. Chem. 2008, 29, 1019-1031.

${ }^{61}$ http://cp2k.berlios.de/ 2000-2012.

${ }^{62}$ Murdachaew, G.; Mundy, C.; Schenter, G.; Laino, T.; Hutter, J. J. Phys. Chem. A 2011, 115, 6046-6053.
${ }^{63}$ Burnham, C.; Xantheas, S. J. Chem. Phys. 2002, 116, 5115.

${ }^{64}$ VandeVondele, J.; Hutter, J. J. Chem. Phys. 2007, 127, 114105.

${ }^{65}$ Becke, A. J. Chem. Phys 1993, 98, 5648.

${ }^{66}$ Stephens, P.; Devlin, F.; Chabalowski, C.; Frisch, M. J. Phys. Chem. 1994, 98, 11623-11627.

${ }^{67}$ Klimes, J.; Michaelides, A. J. Chem. Phys. 2012, $137,120901$.

${ }^{68}$ Chen, W.; Gordon, M. J. Phys. Chem. 1996, 100, 14316-14328.

${ }^{69}$ Shank, A.; Wang, Y.; Kaledin, A.; Braams, B.; Bowman, J. J. Chem. Phys. 2009, 130, 144314.

${ }^{70}$ Mas, E.; Bukowski, R.; Szalewicz, K. J. Chem. Phys. 2003, 118 4386-4403.

${ }^{71}$ Caldwell, J.; Dang, L.; Kollman, P. J. Am. Chem. Soc 1990 , 112, 9144-9147.

${ }^{72}$ Galassi, M. GNU Scientific Library Reference Manual, 3rd ed.; Network Theory Ltd., 2009.

${ }^{73}$ Maple 11, Maplesoft, a division of Waterloo Maple Inc., Waterloo, Ontario

${ }^{74}$ Partridge, H.; Schwenke, D. J. Chem. Phys. 1997, 106, 4618.

${ }^{75}$ Anderson, J.; Crager, K.; Fedoroff, L.; Tschumper, G. J. Chem. Phys. 2004, 121, 11023-11029.

${ }^{76}$ Hill, T. An Introduction to Statistical Thermodynamics; Dover, 1986.

${ }^{77}$ Mayer, J.; Mayer, M. Statistical Mechanics; John Wiley \& Sons Inc, 1940.

${ }^{78}$ Mason, E.; Spurling, T. International encyclopedia of physical chemistry and chemical physics. Topic 10, Fluid state, V. 2; Pergamon Press: New York, 1969.

${ }^{79}$ Harvey, A.; Lemmon, E. J. Phys. Chem. Ref. Data. 2004, 33, 369-376.

${ }^{80}$ Mas, E.; Szalewicz, K. J. Chem. Phys. 1996, $104,7606$.

${ }^{81}$ Benjamin, K.; Singh, J.; Schultz, A.; Kofke, D. J. Phys. Chem. $B$ 2007, 111, 11463-11473.

${ }^{82}$ Kell, G.; McLaurin, G.; Whalley, E. Proc. R. Soc. Lond. A 1989, 425, 49-71.

${ }^{83}$ Garberoglio, G. Chem. Phys. Lett. 2012, 525-526, 19-23.

${ }^{84}$ Schenter, G. J. Chem. Phys. 2002, 117, 6573.

${ }^{85}$ Shaul, K.; Schultz, A.; Kofke, D. J. Chem. Phys. 2011, 135, 124101

${ }^{86}$ Wernet, P.; Nordlund, D.; Bergmann, U.; Cavalleri, M.; Odelius, M.; Ogasawara, H.; Näslund, L.; Hirsch, T.; Ojamäe, L.; Glatzel, P.; Pettersson, L.; Nilsson, A. Science 2004, 304, 995999.

${ }^{87}$ Clark, G.; Cappa, C.; Smith, J.; Saykally, R.; Head-Gordon, T. Mol. Phys. 2010, 108, 1415-1433.

${ }^{88}$ Pieniazek, P.; Tainter, C.; Skinner, J. J. Am. Chem. Soc. 2011, $133,10360$.

${ }^{89}$ Nihonyanagi, S.; Ishiyama, T.; Lee, T.; Yamaguchi, S.; Bonn, M.; Morita, A.; Tahara, T. J. Am. Chem. Soc. 2011, 133, 1687516880 .

${ }^{90}$ Kumar, P.; Franzese, G.; Eugene Stanley, H. J. Phys. Condens. Matter 2008, 20, 244114.

${ }^{91}$ Limmer, D.; Chandler, D. J. Chem. Phys. 2011, 135, 134503. 